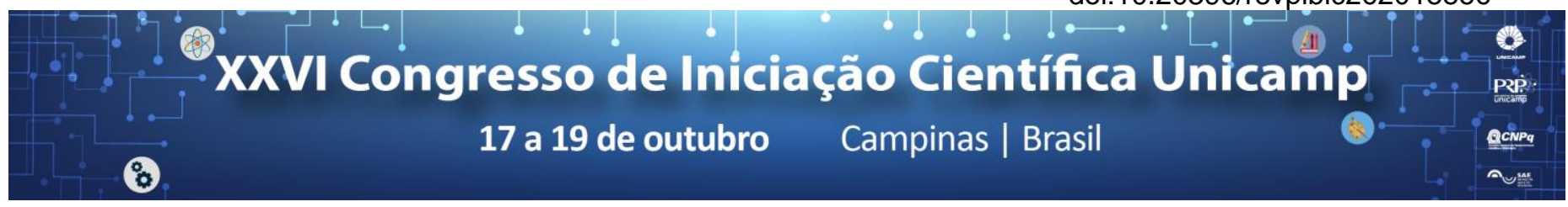

\title{
How do MK-801 and Risperidone Modulate the Proteome of Human Oligodendrocytes?
}

\author{
Caroline B. Teles, Lívia R. da Silva*, Daniel Martins-de-Souza
}

\begin{abstract}
Schizophrenia is a complex neuropsychiatric disorder developed by genetic and environmental factors. The treatment is based on antipsychotics, such as risperidone. Some of illness symptoms has been mimic by MK-801, a non-competitive antagonist of glutamate NMDA receptor. Proteome evidences have been shown disturbances in oligodendrocytes metabolism of schizophrenia patients, mainly in energy metabolism. The aim of this study is to characterize the proteome of a human oligodendrocyte cell line treated with MK-801, risperidone and the co-treatment, in order to shed light about the interactions between schizophrenia and the antipsychotic drug risperidone.
\end{abstract}

Key words: schizophrenia, NMDA receptor, risperidone

\section{Introduction}

Schizophrenia is a multifactorial psychiatric disease that affects $1 \%$ of the world's population. The symptoms include hallucinations, cognitive dysfunction and social isolation; which are treated with antipsychotic, such as risperidone ${ }^{1}$. Strong evidences have been shown the hypofunction of NMDA glutamate receptor and glutamatergic dysfunction in patients with schizophrenia ${ }^{2}$. MK-801, a NMDA receptor antagonist, has been employed in cell culture to mimic this dysfunctions in glutamate neurotransmission. Thus, it has been considered as a model for some aspects of illness ${ }^{3}$.

Besides alterations in neurotransmission, the loss of oligodendrocytes as well as the decrease of myelination in prefrontal cortex of schizophrenia patients have been reported ${ }^{4}$. These cells are involved in myelination process and axonal energy supply on central nervous system. Proteome analysis of post mortem brain tissue have been demonstrated oligodendrocytes dysfunctions in schizophrenia patients, mainly in glycolytic pathways ${ }^{5}$. The aim of this study was to investigate the biological processes and metabolic pathways involved in the treatment with risperidone, MK801 and the co-treatment in a human oligodendrocytes cell line (MO3.13).

\section{Results and Discussion}

MO.313 cells were cultured, subsequently treated with risperidone, MK801 and co-treatment (MK801 plus risperidone after $4 \mathrm{~h}$ ), and collected after $8 \mathrm{~h}$. Proteins were extracted, digested and proteomic analyses were performed using mass spectrometry. Data were processed by the Progenesis software, where the proteins were identified and quantified. The differentially expressed proteins ( $p$-value $<0,05)$ were analysed using bioinformatics tools available online; DAVID (https://david.ncifcrf.gov) and PANTHER (www.pantherdb.org).

Compared to control, in MK-801 treatment we identified 1250 proteins, of these 379 were differentially expressed. These proteins were mainly related with cellular process, metabolic process and cellular component organization or biogenesis. About metabolic pathways, these proteins were associated with ribosome, spliceosome and carbon metabolism. Risperidone treatment, we identified 1708 proteins, in which 197 have their expression altered. These proteins mainly play role in cellular process, metabolic process and cellular component organization or biogenesis. The main pathways affected include ribosome and spliceosome metabolism. For the cotreatment (MK801+Risperidone) we identified 1250 proteins wich 88 were present at different levels. Most of these proteins were related with cellular process, metabolic process and cellular component organization or biogenesis. These proteins participated at some metabolic pathways such as ribosome, spliceosome and RNA transport.

\section{Conclusions}

Proteome studies allows the analysis of differentially expressed proteins that play a key role in risperidone treated oligodendrocytes. The data obtained can provide a better understanding about the biological process and metabolic pathways altered by this drug as well as its efficacy. However, more discussion about the results are necessary.

${ }^{1}$ Woodward ND, E. al. A meta-analysis of neuropsychological change to clozapine, olanzapine, quetiapine, and risperidone in schizophrenia. PubMed - NCBI https://www.ncbi.nlm.nih.gov/pubmed/15784157 (accessed Jul 3, 2018).

${ }^{2}$ Weickert, C. S.; Fung, S. J.; Catts, V. S.; Schofield, P. R.; Allen, K. M.; Moore, L. T.; Newell, K. A.; Pellen, D.; Huang, X.-F.; Catts, S. V.; et al. Molecular Evidence of N-Methyl-D-Aspartate Receptor Hypofunction in Schizophrenia. Mol. Psychiatry 2013, 18, 1185-1192.

${ }^{3}$ Brandão-Teles, C.; Martins-de-Souza, D.; Guest, P. C.; Cassoli, J. S. MK-801-Treated Oligodendrocytes as a Cellular Model to Study Schizophrenia. In Proteomic Methods in Neuropsychiatric Research; Springer, Cham, 2017; pp. 269-277.

${ }^{4}$ Uranova, N. A.; Vostrikov, V. M.; Orlovskaya, D. D.; Rachmanova, V. I. Oligodendroglial Density in the Prefrontal Cortex in Schizophrenia and Mood Disorders: A Study from the Stanley Neuropathology Consortium. Schizophr. Res. 2004, 67, 269-275

${ }^{5}$ Martins-de-Souza D, E. al. Proteome analysis of the thalamus and cerebrospinal fluid reveals glycolysis dysfunction and potential biomarkers candidates for schizophrenia. - PubMed - NCBI https://www.ncbi.nlm.nih.gov/pubmed/20471030 (accessed Jul 5, 2018) 